\title{
Analysis of Computational Models to Describe Individual Decision-Making Process
}

\author{
Eugene Santos Jr \\ Thayer School of Engineering \\ Dartmouth College, Hanover, \\ New New Hampshire, USA, \\ Eugene.Santos.Jr@dartmouth.edu \\ Hien Nguyen \\ Department of Computer Science \\ University of Wisconsin - \\ Whitewater, Whitewater, \\ Wisconsin, USA, \\ nguyenh@uww.edu
}

\author{
Keum Joo Kim \\ Thayer School of Engineering \\ Dartmouth College, Hanover, \\ New New Hampshire, USA, \\ Keum.Joo.Kim@dartmouth.edu \\ Luke Veenhuis \\ Department of Computer Science \\ University of Wisconsin - \\ Whitewater, Whitewater, \\ Wisconsin, USA, \\ veenhuislj12@uww.edu
}

\author{
Jacob A Russell \\ Thayer School of Engineering \\ Dartmouth College, Hanover, \\ New New Hampshire, USA, \\ Jacob.A.Russell@dartmouth.edu \\ Luke Deguelle \\ Department of Computer Science \\ University of Wisconsin - \\ Whitewater, Whitewater, \\ Wisconsin, USA, \\ deguellelt29@uww.edu
}

\begin{abstract}
Understanding the human decision-making process and evaluating the quality of these decisions has been the focus of many researchers. Previously, we proposed a computational, cognitive framework called the Double Transition Model (DTM) to study human decision-making processes. We applied it to simulate a couple of scenarios developed through a naval warfare simulation game called Steel Ocean. This framework concentrated on the cognitive process of an individual's decision-making process and capturing his cognitive style. One of the key functionalities of this framework has been to provide a reward distribution indicating the quality of decisions made under certain conditions. In this paper, we present a rigorous investigation of our models capturing individual characteristics with respect to decision-making style and the reward distributions. In particular, our models explored the following questions: 1) whether individual models are different from each other like human beings are; 2) whether these models exhibit particular decision-making styles; and 3) whether these models can capture different situations as human beings do. We evaluated the capability of our models capturing these individuals' characteristics by comparing multiple DTMs against each other, each built from a couple of individuals under various circumstances. We confirmed that individual characteristics could be captured in the DTMs. Furthermore, we compared individuals' trajectories (i.e., a sequence of decisions) identified
\end{abstract}

Permission to make digital or hard copies of all or part of this work for personal or classroom use is granted without fee provided that copies are not made or distributed for profit or commercial advantage and that copies bear this notice and the full citation on the first page. Copyrights for components of this work owned by others than ACM must be honored. Abstracting with credit is permitted. To copy otherwise, or republish, to post on servers or to redistribute to lists, requires prior specific permission and/or a fee. Request permissions from permissions@acm.org.

WI '19, October 14-17, 2019, Thessaloniki, Greece

(c) 2019 Association for Computing Machinery.

ACM ISBN 978-1-4503-6934-3/19/10...\$15.00

https://doi.org/10.1145/3350546.3352515 by multiple DTMs in addition to their associated neighbors to verify that decision-making process in various social conditions could be described with DTMs. Our empirical study was conducted on two sets of real-world data: Supervisory Control Operations User Testbed (SCOUT) and the naval warfare simulation game (Steel Ocean).

\section{KEYWORDS}

Decision-making process, cognitive style, computational framework, naval warfare, supervisory control operation.

\section{ACM Reference format:}

Eugene Santos Jr, Keum Joo Kim, Jacob A Russell, Hien Nguyen, Luke Veenhuis and Luke Deguelle. 2019. Analysis of Computational Models to Describe Individual Decision-Making Process. In Proceedings of WI '19: IEEE/WIC/ACM International Conference on Web Intelligence (WI '19), October 14-17, 2019, Thessaloniki, Greece. ACM, New York, NY, USA, 8 pages. https://doi.org/10.1145/3350546.3352515

\section{Introduction}

How do individuals differ in arriving at decisions? How come someone can arrive at better decisions than others? Can somebody identify the right decisions faster than others? If so, do they have any habitual preferences for making such decisions? These questions have been asked by many scientists, and they found that individuals have habitual tendencies to make decisions in a consistent manner, called decision-making styles, which vary among individuals $[1,2]$.

Previously, we had proposed a computational, cognitive framework, called the Double Transition Model (DTM) [3] and developed the theory into practice by modeling real commanders in a naval warfare simulation game (Steel Ocean). Each commander's cognitive style had been identified through modeling his decision-making processes based on the information gathered from his perceptions and observations. 
These models and their analysis had been validated by comparing those of theoretical users of canonical decisionmaking styles against each other: rational, intuitive, and spontaneous styles. Specifically, we had identified significant properties of graphical structure and reward distribution of commanders by analyzing their computational models demonstrated by their cognitive processes alongside the information they collected during the task [4]. We also performed analysis on unmanned aerial vehicle (UAV) operators performing tasks in the Supervisory Control Operations User Testbed (SCOUT) [5], developed at the US Naval Research Laboratory. Leveraging SCOUT and the Steel Ocean results, we identified the information contained within trajectories (i.e. sequences of decisions described in the DTMs) reflecting individual differences observed from the real-world SCOUT records and unique properties of the DTMs representing human commanders in Steel Ocean using k-means clustering [6].

There have been other state of the art approaches using machine learning to solve decision-making processes such as in playing Go [12], but they decide differently than humans do [13]. The closest research related to our work is adapting computergenerated recommendations for personalization in interactive narrative systems [14]. Like the Alpha Zero approach, however, it requires all of the rules to be coded a priori to simulate the environment.

In this paper, we expand the original study further with SCOUT and the human commanders playing Steel Ocean by addressing mixed decision-making styles. We identified the graphical properties of DTMs from the human commanders with the help of a psychological instrument and derived individual decision-making styles for SCOUT subjects. We observed that the commander with a spontaneous and avoidant decisionmaking style is more persistent than the commander with a rational style.

In addition, we focus on individual characteristics by emphasizing risk-aversion, selection utility, and game performance. We examined the correlation between the individual characteristics identified from the original raw data and the trajectory statistics obtained from DTMs and identified that some statistics could demonstrate individual characteristics more effectively than others. Furthermore, we compared the trajectory statistics of individuals in multiple DTMs and confirmed that our models could describe the decision-making process in various social conditions.

This paper is organized as follows. First, we review the prior studies pertinent to decision-making styles, individual differences, and computational cognitive models for human decision-making processes. Second, we describe three technical components of our overarching framework: Double Transition Model (DTM), dynamic Markov Decision Process (dMDP) [7] and Inverse Reinforcement Learning (IRL) [8]. Lastly, we assess the applicability of our theoretical framework against the realworld datasets described above and present our conclusions

\section{Related Work}

Commanders are individuals with a responsibility to make critical decisions on the battlefield. Our goal is to help them make effective and efficient decisions, which is critical to humanitarian benefits in addition to the financial gains. Therefore, understanding the human decision-making process and identifying unique decision-making styles and characteristics has been a fundamental part of our research. In this section, we review some previous studies for decisionmaking styles, individual characteristics, and computational cognitive models.

\subsection{Decision-Making Style}

Individuals use their habitual patterns in making decisions since they perceive circumstances, gather varying amounts of information, consider multiple alternatives in their ways, and respond in diverse manners. The habitual patterns individuals apply in making sense of the data they collected while deriving decisions have been defined in various decision-making styles. For instance, decision-making style has been defined as "the learned habitual response pattern exhibited by an individual when confronted with a decision situation" and "individuals" characteristic mode of perceiving and responding to decisionmaking tasks" [1,2]. Decision-making styles can describe how to search for information, how many alternatives to consider, and how to evaluate those alternatives. Among the original five decision-making styles proposed in [2], we address three canonical decision-making styles here: rational, intuitive, and dependent. A rational style conducts a comprehensive search for information, keeps a significant number of alternatives, and logically evaluates them. An intuitive style pays attention to details rather than a systematic search for information, relies on previous experience, and evaluates emotionally. A dependent style searches for advice and guidance from others and follows it for evaluating alternatives.

\subsection{Individual Characteristics}

Although there can be an unlimited number of attributes demonstrating human behaviors, here we address only three: risk-aversion, selection utility, and game performance for our analysis since they are well supported as key components in making decisions and feasible to obtain from our dataset [5,9].

Risk-aversion is known as a fundamental component of decision-making behaviors [9], but its analysis is frequently challenged by the lack of accurate measurement and consistency against time. Selection utility has been well studied for many decades as a key component of human behaviors. Utility can explain the usefulness of decision alternatives and predict how much the information can be used for decision-making. Game reward (performance) encourages players to implement different strategies by providing positive feedback and boosts selfconfidence. Although the theory of self-determination and cognitive evaluation warns the side effect of deteriorating internal motivations, its benefit of controlling game habits and disorders have been frequently reported. 


\subsection{Computational Cognitive Models}

Computational cognitive models have been developed in multiple ways while focusing on spatial memory representing knowledge. Due to the challenges associated with sensory noise, uncertainty, and high-dimensional complexity, Bayesian localization has been preferred in recent studies [10, 11].

Models based on spatial knowledge representation can be classified into symbolic, neural network, and cognitive architectures depending on the level of specificity, type of representation scheme, structural accuracy, generalization and abstraction, and dimensional complexity. Symbolic models describe knowledge relying on explicit logic rules and operate on a high-level of abstraction and function more effectively than neural network models. In contrast, neural network models utilize a number of simple processing units affecting each other via weighted connections, operate in parallel, employ distributed representations, and learn rules from training data instead of encoding explicit rules. Therefore, neural networks can perform well for complex tasks. Since the ultimate goal of cognitive models is to demonstrate a wide range of cognitive phenomena and to simulate multiple cognitive mechanisms, recent research incorporates the functionality of the symbolic models and complexity of the neural networks.

\section{Our Approach}

Our approach is an overarching computational framework to describe the human decision-making process. The foundation of our study is the Double Transition Model (DTM) developed for modeling the formation and change of human opinions [3]. In this section, we provide a summary of the DTM in addition to two other theoretical and technical ingredients embedded in our system. We employed the Markov Decision Process (MDP) [7] as a way to connect an individual's cognitive states and Inverse Reinforcement Learning to identify reward distributions pertinent to decisions made by individuals.

\subsection{Double Transition Model (DTM)}

The DTM is a cognitive, computational model, which has been used to capture human opinion formation and change [3]. We use a DTM to capture human decision-making processes in which each node represents a cognitive state of an individual, and each edge represents the transitions between two cognitive states. The transition between two states happens when an individual takes an action that moves his current cognitive state to the new one. Each node in a DTM is composed of two subgraphs, a Query Transition Graph (QTG) and a Memory Transition Graph (MTG). A node in a QTG describes a single query with one targeted random variable of interest and a set of other known or unknown random variables representing the information relevant to the observation and perception available at that time to that individual. We build a DTM from a set of episodic tasks of an individual, which consists of a set of actions, and observations of environmental settings to construct a set of cognitive states and transitions between them. These actions and cognitive states form the basis of a trajectory. From a DTM, we can learn a reward distribution to arrive at the reward value assigned to each cognitive state at a given time point.

\subsection{Markov Decision Process (MDP)}

The DTM can capture the individual's actions and his cognitive states and even models how these change over time. However, the DTM does not know which of the individual' cognitive states are preferred. Therefore, we relied on a Markov Decision Process (MDP) [7] to determine the cognitive state preferred over other competing states. The original Markov models have been generalized into Markov decision processes and utilized for describing human decision-making processes over time. MDPs have been successfully utilized in a variety of fields including robotics for indoor robot navigation problems, medical decisionmaking, and planning for target selection in military decisionmaking processes. A discrete-time MDP $\mathscr{M}$ can be described by a set of finite number of states $(S)$, a set of actions/decision $(A)$, a transition probability for each pair of states $(P)$, a set of rewards/cost $(R)$, and a discount factor $(\gamma)(S, A, P, R, \gamma)$. In addition, there are two more important components in MDPs, which are a decision rule specifying possible actions given state and optimal policy identifying a sequence of decisions with maximum expected rewards.

A DTM could be mapped to an MDP at any given time, but a single MDP is not sufficient to describe the change of an individual's decisions. This problem becomes more serious when we describe the change of decisions associated with the learning effect. Therefore, we generate new MDPs to account for any new, dynamic information and call these generated MDPs dynamic Markov Decision Processes (dMDPs) because they are generated on-the-fly. With this, our model can address that the process of decision-making is dynamic with a steady flow of incoming and outgoing information streams

\subsection{Inverse Reinforcement Learning (IRL)}

Inverse Reinforcement Learning (IRL) was developed to determine a reward function that can explain observed, optimal behavior [8]. Our scheme to derive reward distribution differs from the original formulation. Our challenges include 1) the Markov property; 2) interdependence of feature values; 3) interdependence of the meaning of rewards. First, when we model individuals' decision-making processes, it is not guaranteed that the conditional probability distribution of an individual's future cognitive state depends only upon the present state. Second, the feature variable-value pairs are selected according to their importance in decision-making processes or associated algorithmic criteria applied, but the original algorithm assumes that features are correlated in a linear function. Lastly, it is not clear how to assess the quality of individuals' decisions and evaluate them properly through reward function when the policy space is incomplete and based on uncertain information. Unfortunately, we found that the linearity of reward distribution can be contradictory to the nonlinear property of the human 
decision-making process. Therefore, we developed a new IRL algorithm addressing the followings:

- $\quad$ The space of trajectories under our consideration is not complete but reflects an individual's observations. The rest of the space must be interpolated or extrapolated for modeling an entire cognitive space.

- A linear combination of states (or features) cannot determine rewards directly because there are likely more complicated relationships between the features in real situations.

- The distribution of trajectory rewards cannot be generalized directly since the trajectory probability varies depending on the other conditions.

- In a trajectory, the probability of occurrence of cognitive states, with respect to features, may not be associated with rewards, but relevant to inevitability.

- $\quad$ Trajectories are neither optimized nor ordered.

- A decision-maker prefers some trajectories to others.

\section{Analysis}

The goal of our evaluation is to capture the differences among individuals by analyzing their corresponding DTMs. Our first dataset was collected from university students playing Commanders in a naval warfare simulation game named Steel Ocean. The second dataset is the Supervisory Control Operations User Testbed (SCOUT) [5] developed by the US Naval Research Laboratory. To evaluate DTMs based on our framework, we gathered two sets of DTMs, a set of local DTMs that represents only one individual's trajectories and a global DTM having trajectories of all individuals with the same goal, and compared them against each other.

While the information about the decision-making styles was collected directly from subjects playing the Steel Ocean by conducting a psychological test on those subjects, the information was not available from SCOUT. Therefore, we investigated the properties of the DTMs built from individual subjects playing Steel Ocean first and leveraged this finding to understand the Scout participants.

\subsection{Human Commanders}

The human Commanders testbed refers to a set of Naval war game run-through played by a team of three students at UWWhitewater. The game is called Steel Ocean ${ }^{1}$ and is selected because of its availability and its community of online users. Each game consists of a set of episodic traces which help us to understand the Commanders' decision-making process. We denoted our Commanders as C1, C2, and C3, respectively. Local

\footnotetext{
${ }^{1}$ Steel Ocean is available at

http://store.steampowered.com/app/390670/
}

DTMs were constructed from C1, C2, and C3 separately, while a global DTM was built from all the records of Commanders involved in Steel Ocean. The numbers of trajectories embedded in these local DTMs were 16,8 , and 8 respectively, while the global DTM had 32 trajectories inside.

4.1.1 Selected Features. We collected a set of 5 features, as shown in Table 1 to construct a trajectory. Each feature is measured as a real value (TD/ETD, ES/FS, TS/ETS, BD) number or as an integer (Damage Spread).

Table 1: Features selected for Trajectories

\begin{tabular}{|l|l|}
\hline $\begin{array}{l}\text { Feature } \\
\text { name }\end{array}$ & Description \\
\hline TD/ETD & $\begin{array}{l}\text { The ratio between team damage (TD) and } \\
\text { enemy team damage (ETD): TD is the } \\
\text { combined damage of ships led by the } \\
\text { Commander and two supporting staff } \\
\text { while ETD is the combined damage of all } \\
\text { the enemy ships. }\end{array}$ \\
\hline ES/FS & $\begin{array}{l}\text { The ratio between enemies sunk (ES) and } \\
\text { friendlies sunk (FS): ES is the combined } \\
\text { number of enemies sunk by the } \\
\text { Commander and the supporting staff while } \\
\text { FS is the combined death of the ships led } \\
\text { by the Commander and the support staff. }\end{array}$ \\
\hline $\begin{array}{l}\text { Damage } \\
\text { (with } \mathrm{n} \text { in the } \\
\text { range of 1000- } \\
5000)\end{array}$ & $\begin{array}{l}\text { Damage spread indicates the number of } \\
\text { people (of the Commander and supporting } \\
\text { staff) who did at least } n \text { damage to the } \\
\text { enemy team. }\end{array}$ \\
\hline TS/ETS & $\begin{array}{l}\text { The ratio between team score and enemy } \\
\text { team score: our team score is the } \\
\text { combined game score of the Commander } \\
\text { and supporting staff. }\end{array}$ \\
\hline BD(s) & $\begin{array}{l}\text { Battle duration is the time (in minutes) } \\
\text { that a run-through took. }\end{array}$ \\
\hline
\end{tabular}

4.1.2 Decision-making style. We determined each individual decision-making style using an instrument of general decisionmaking style, which consists of 25 questionnaires [2]. A set of answers associated with the five questions was used for each style, and each answer is rated on a scale from 1 to 5 ( 1 is the lowest, and 5 is the highest). We tallied the points from each group of questions with respect to each style and determined the final style being the ones with highest scores. The results were presented as DMS (decision-making style) in Table 2 below. The performance denotes the ratio between win and loss for each Commander. To analyze the graphical properties of DTMs, we considered the number of states, actions, density, and their connectivity. We computed density D as follows:

$$
D=\frac{|E|}{|V|(|V|-1)}
$$

$|V|$ denotes the unique number of cognitive actions and states, and $|E|$ represents all in (out) degrees associated with the cognitive states and actions. The state degree $[\mathrm{D}($ State $)]$ and the 
Analysis of Computational Models to Describe Individual DecisionMaking Process

action degree $[\mathrm{D}$ (action)] were computed by getting the sum of in and out-degree of actions and states

Table 2: Graphical Properties

\begin{tabular}{|l|l|l|l|}
\hline Commander & C1 & C2 & C3 \\
\hline DMS & Rational & $\begin{array}{l}\text { Spontaneous } \\
\text { \& Avoidant }\end{array}$ & $\begin{array}{l}\text { Rational } \\
\text { \& Dependent }\end{array}$ \\
\hline Performance & 0.667 & 0.33 & 0.5 \\
\hline State & 256 & 116 & 94 \\
\hline Action & 199 & 82 & 73 \\
\hline Density & $1.10 \mathrm{E}-02$ & $2.73 \mathrm{E}-02$ & $2.93 \mathrm{E}-02$ \\
\hline D(State) & 1574 & 742 & 564 \\
\hline D(Action) & 690 & 324 & 248 \\
\hline
\end{tabular}

4.1.3 Trajectory Statistics. As mentioned in Table 2, $\mathrm{C} 1$ is a rational decision-maker with 16 trajectories, $\mathrm{C} 2$ is spontaneous and avoidant with 8 trajectories, and $\mathrm{C} 3$ is rational and dependent with 8 trajectories. From all of the trajectories belonging to each Commander, we computed the minimum, maximum, average, and standard deviation of trajectory probability and sum of rewards from local and global DTMs for each Commander.

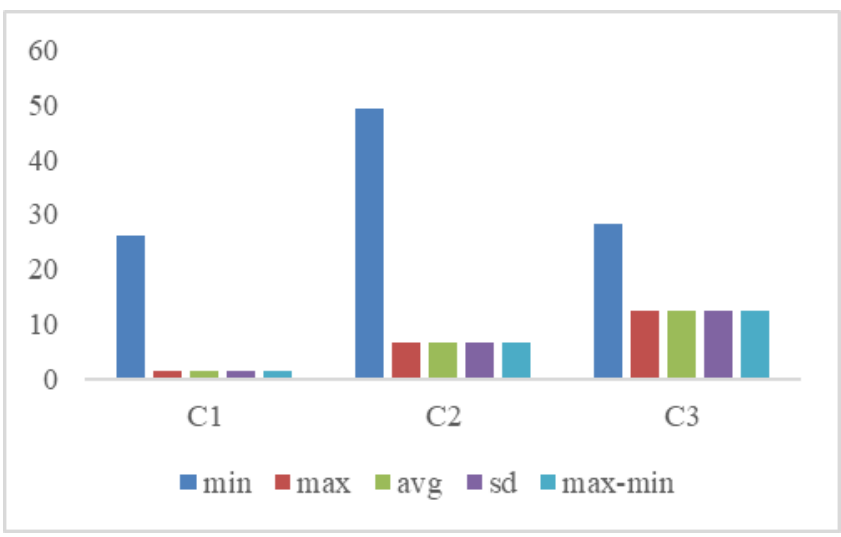

Figure 1: Trajectory Probabilities in local/global DTMs

Trajectory probability cannot be an absolute measure of granularity of DTMs but can be a good indicator of how likely the trajectories would be taken by the individual. In local DTMs, the trajectory probabilities of $\mathrm{C} 1$ were spread from $2.88 \mathrm{E}-72$ to 4.17E-22 with AVG (5.10E-23) and SD (1.39E-22), that of C2 were from $6.78 \mathrm{E}-36$ to $1.33 \mathrm{E}-16$ with AVG (1.67E-17) and SD (4.68E17 ), and that of $\mathrm{C} 3$ were from $4.72 \mathrm{E}-23$ to $1.03 \mathrm{E}-07$ with $\mathrm{AVG}$ (1.29E-08) and SD (3.63E-08). The difference between maximum and minimum was observed as $(4.17 \mathrm{E}-22,1.32 \mathrm{E}-16,1.027 \mathrm{E}-07)$ for $\mathrm{C} 1, \mathrm{C} 2$, and $\mathrm{C} 3$, respectively. Therefore, we observed that $\mathrm{C} 1$ has the widest range of trajectory probabilities. In a sense, this is natural since $\mathrm{C} 1$ has the most trajectories, but $\mathrm{C} 2$ has a wider range than $\mathrm{C} 3$ despite the same number of trajectories. In the
WI '19, October 14-17, 2019, Thessaloniki, Greece

global DTM, the trajectory probability of C1 were spread from $1.595 \mathrm{E}-98$ to $2.08 \mathrm{E}-23$ with AVG (1.30E-24) and SD (5.205E-24), that of C2 were from $3.92 \mathrm{E}-85$ to $2.39 \mathrm{E}-23$ with AVG (3.00E-24) and SD (8.46E-24), and that of C3 were from $2.077 \mathrm{E}-51$ to $3.56 \mathrm{E}-$ 20 with AVG (4.44E-021) and SD (1.257E-20). The difference between maximum and minimum was observed as $(2.08 \mathrm{E}-23$, 2.39E-23, 3.56E-20) for $\mathrm{C} 1, \mathrm{C} 2$, and $\mathrm{C} 3$ respectively. Unlike the local DTMs, the range of trajectory probabilities of $\mathrm{C} 1$ and $\mathrm{C} 2$ in the global DTM was scaled similarly while that of $\mathrm{C} 1$ is smaller than the others. Figure 1 represents the logarithm of the ratio of each statistic of trajectory probability obtained from local and global DTMs. Although the ratio of minimum values of $\mathrm{C} 2$ is the largest (49.24) while that of $\mathrm{C} 1$ is the smallest (26.26). For other statistics, those of $\mathrm{C} 1$ are the smallest, while those of $\mathrm{C} 3$ are the largest consistently.

The sum of the rewards (R) of each trajectory is a key measure to assess the fitness of the trajectory under the circumstances. In local DTMs, R of all trajectories C1 were spread from [-2700, 0] with AVG (-887.5) and SD (741.97), that of C2 were from -3200 to -300 with AVG $(-1500)$ and SD (988.5), and that of C3 were from -4500 to -1400 with AVG (-2700) and SD (938.08). The difference between maximum and minimum was observed as $(2700,2900,3100)$ for C1, C2, and C3, respectively. In the global DTM, R of all trajectories C1 were spread from -1100 to 0 with AVG (-375) and SD (329.65), that of C2 were from -1600 to -300 with AVG (-725) and SD (562.52), and that of C3 were from -700 to 0 with AVG (-350) and SD (302.37). The difference between maximum and minimum was observed as (1100, 1600, 700) for $\mathrm{C} 1, \mathrm{C} 2$, and $\mathrm{C} 3$ respectively. In general, $\mathrm{R}$ values obtained from local DTMs were turned out to be bigger than those obtained from the global DTM.

\subsection{Supervisory Control Operations Testbed}

The SCOUT dataset was collected from the US Naval Research Laboratory. 20 volunteer human subjects executed pre-scripted Unmanned Aerial Vehicle (UAV) missions under the Supervisory Control Operation User Testbed [5]. The participants controlled multiple heterogeneous UAVs while earning (or losing) points during a twenty-minute long supervisory control mission. They joined the operation in two consecutive sessions, and the records of their decisions were used for two trajectories. Therefore, 20 local DTMs were established, and each DTM is composed of two trajectories, while a global DTM was established from 40 trajectories belonging to 20 participants. They were rewarded once they located the appropriate vehicle within the range of target sensors and penalized when violating restricted airspace rules. They could get more rewards by responding to chat messages rapidly and correctly.

4.2.1 Selected Features. A set of 9 features was selected shown in Table 3, which were identified as important out of 194 features through recursive partitioning and tree-based methods. For attributes having continuous values, we discretized them into 5 levels. Due to the sparsity of the original data, we extracted the original observations only when the value of "ScoreChange" was meaningful (i.e., neither NA nor empty), and missing values 
were substituted by the observables collected at the closest timestamp.

Table 3: SCOUT Features selected

\begin{tabular}{|l|l|}
\hline Feature name & Description \\
\hline MsgOut & $\begin{array}{l}\text { Participant's written responses to } \\
\text { messages in the Simulation's chat } \\
\text { boxes }\end{array}$ \\
\hline SE_EstDelay & $\begin{array}{l}\text { Estimated delay across the } \\
\text { network }\end{array}$ \\
\hline RtPupilDiameter & $\begin{array}{l}\text { Right pupil diameter size, not } \\
\text { accounting for quality of the data }\end{array}$ \\
\hline Asset2_NDdist & $\begin{array}{l}\text { UAV's distance to the next } \\
\text { destination }\end{array}$ \\
\hline LtEyeLidOpeningQ & $\begin{array}{l}\text { Quality of the left eyelid data from } \\
\text { SmartEye }\end{array}$ \\
\hline eventType & $\begin{array}{l}\text { Marks when an event occurs in } \\
\text { the simulation- both when a } \\
\text { scripted event occurs and when } \\
\text { the user performs an event }\end{array}$ \\
\hline RtFILTPupilDiam & $\begin{array}{l}\text { A quality weighted average filter } \\
\text { for the right pupil size }\end{array}$ \\
\hline ScoreChange & $\begin{array}{l}\text { Shows when feedback was } \\
\text { provided to the user about points } \\
\text { that they have earned }\end{array}$ \\
\hline Action & $\begin{array}{l}\text { Shows more detailed information } \\
\text { about what action the user took in } \\
\text { response to an event }\end{array}$ \\
\hline
\end{tabular}

4.2.2 Individual Characteristics. For risk-aversion, we summed up the number of restricted region violations of individuals during the two consecutive sessions and computed the average. For selection utility, we computed the average repeated occurrence of feature values associated with action, event, and chat messages. For game performance, we summed up all changes in scores for each individual during each session and computed the average. For selection utility and game performance, we normalized the original value against the average value.

In Figure 2 , the $\mathrm{x}$-axis denotes the participant ID, and the $\mathrm{y}$ axis represents risk-aversion, game performance, and selection utility explained above. We observed P6, P8, and P19 violated the region restriction most frequently while $\mathrm{P} 3, \mathrm{P} 4, \mathrm{P} 9, \mathrm{P} 10, \mathrm{P} 14, \mathrm{P} 16$, $\mathrm{P} 21, \mathrm{P} 22$, and P23 did not violate the rule at all. Regarding the game performance, P3 was observed to get the highest average game score (12075) of all, while P8 got the lowest average score (7225). The average of all 20 participants' game score was 10132.5. Therefore, the game performance described for P3 is 1.192, and that for P8 is 0.713 in Figure 1 . With respect to selection utility, P15 showed the highest value (17.41) and P14 showed the lowest utility while the average utility of all was identified as 15.69 .

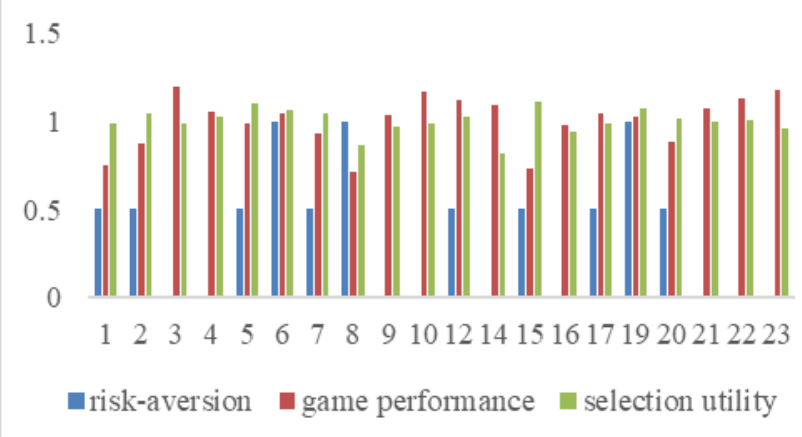

Figure 2: Individual Characteristics

4.2.3 Decision-making style. In order to infer the decisionmaking styles of SCOUT participants, we analyzed the graphical properties of DTMs by comparing their statistics. Decisionmakers in the real world might not be simply classified into any canonical decision-making style. By comparing our earlier analysis of human Commanders, we observed that the structure of DTMs built from decision-makers with rational cognitive style is loosely connected compared against DTMs of spontaneous and avoidant decision-makers. According to the density information obtained from the SCOUT DTMs as shown in Figure 3, we assessed that P19 (1.23E-02) was the closest to the rational decision-making style while P20 (2.51E-02) was the closest to the spontaneous and avoidant cognitive style. The average density of all DTMs is $1.58 \mathrm{E}-02$, and $7 / 20$ was higher than the average.

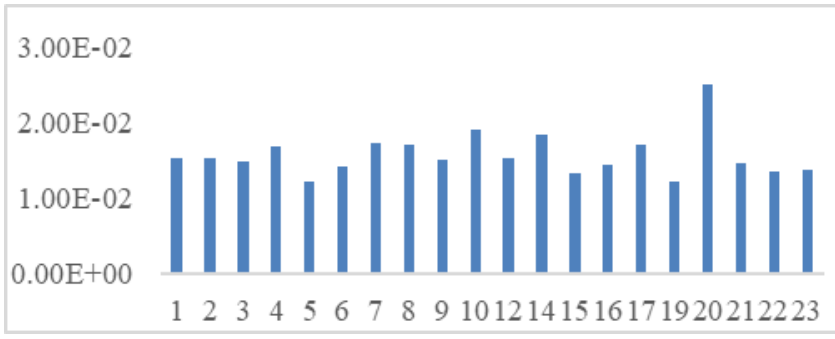

Figure 3: Density of individual DTMs

Regarding the two individuals (P19, P20), we revisited their characteristics and examined whether they were associated with each other in any respect. P20 had the highest density of DTM, which was associated with the spontaneous and avoidant decision-making style. With respect to the individual characteristics, he had medium-level risk-aversion, gameperformance, and selection utility. P19 had the lowest density DTM and was the closest to the rational decision-making style. He violated the restricted region the most, and his selection utility and game performance are above the average.

4.2.4 Trajectory Statistics. The global DTM should be more complex than local DTM due to the diverse range of actions and cognitive states under consideration in general. By comparing the global DTM against local DTMs, we tried to identify the unique individual cognitive process in multiple environments. 


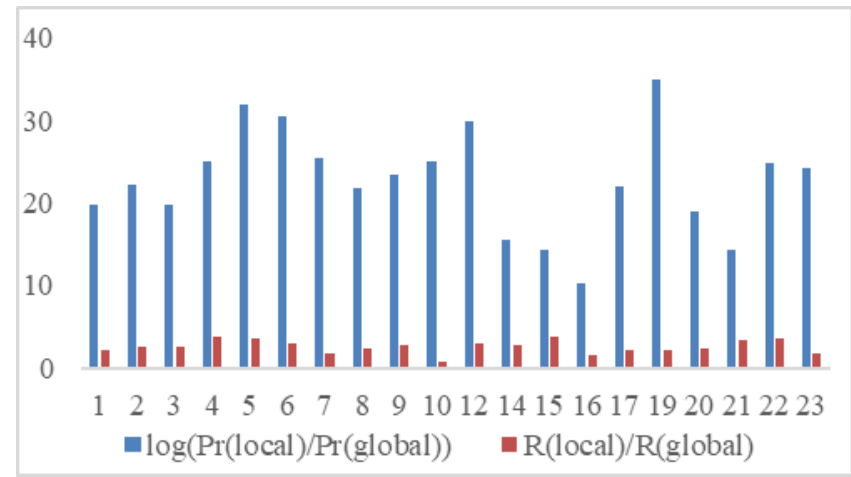

\section{Figure 4: Trajectory statistics in global vs. local DTMs}

A DTM's granularity can be assessed by the trajectories' probabilities. The min and max trajectory probability in the global DTM was observed from $4.32 \mathrm{E}-76$ to $3.01 \mathrm{E}-15$ with the average $(7.52 \mathrm{E}-17)$ while the min and max trajectory probability in local DTMs was identified from $3.64 \mathrm{E}-32$ to $5.75 \mathrm{E}-05$ with the average (1.75E-06). Therefore, we confirmed that the global DTM had a higher granularity than local DTMs. In Figure 4, the level of granularity change between local and global DTMs was presented. For comparing the trajectory probabilities, we used the logarithm of the ratio between local and global trajectory probability (i.e., $\log (\operatorname{prob}($ local $) / \operatorname{prob}($ global $)))$ while the ratio between local and global reward sum was compared directly. The biggest granularity change associated with the trajectory probability was observed from P16 (34.92), while the smallest change was identified as P14 (10.28) with the average (22.85). Regarding the change of trajectory rewards, we observed that P10 showed the smallest (0.77) and P13 showed the biggest (3.88) with the average (2.61).

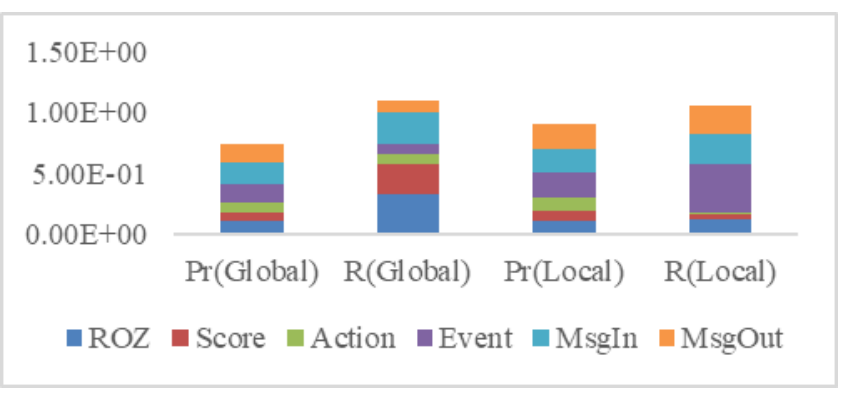

Figure 5: Correlation between Trajectory statistics vs. Individual characteristics

We expected to observe some relevance between individual characteristics (presented in Figure 2) and the trajectory statistics obtained from local and global DTMs (shown in Figure 4). Therefore, we computed the correlation between the feature values considered for individual characteristics and trajectory statistics and summed up the absolute values according to each trajectory statistics as shown in Figure 5. The left-most histogram represents the correlation between trajectory probability in global DTM and a set of the feature values denoting ROZ (restricted region violation), game score, action, event, msgIn, and msgOut. The sum of the correlation between trajectory probability in global DTM and the individual characteristics turned out to be 0.742 . The correlation between the sum of reward in global DTM, the trajectory probability in local DTM, and the sum of reward in local DTM, and the individual differences were observed as 1.1, 0.906 and 1.06 respectively as presented in Figure 5. The sum of trajectory reward in global DTM was identified as the best to demonstrate the individual characteristics.

4.2.5 Neighbors \& Distances in DTMs. In addition to the overall granularity change between global and local DTMs, the number of neighbor trajectories identified can be another key to tell about individuals since they are the potential trajectories with a constant number of differences in actions and states. Here, we considered the neighbor trajectories having only two different actions or states together. We collected the number of neighbor trajectories identified for each individual in global and local DTM and compared them. P14 had the smallest number of neighbor trajectories (121), and P12 had the largest number of neighbors (269) while the average number of neighbors was 186.1 in global DTM. P14 has the smallest number of neighbors (49) while P12 has the largest number of neighbors (180), while the average number of neighbors is 95.65 in local DTMs. It is clear that all individuals have more neighbor trajectories in the global DTM than in local DTMs, but the number of increasing neighbors varies.

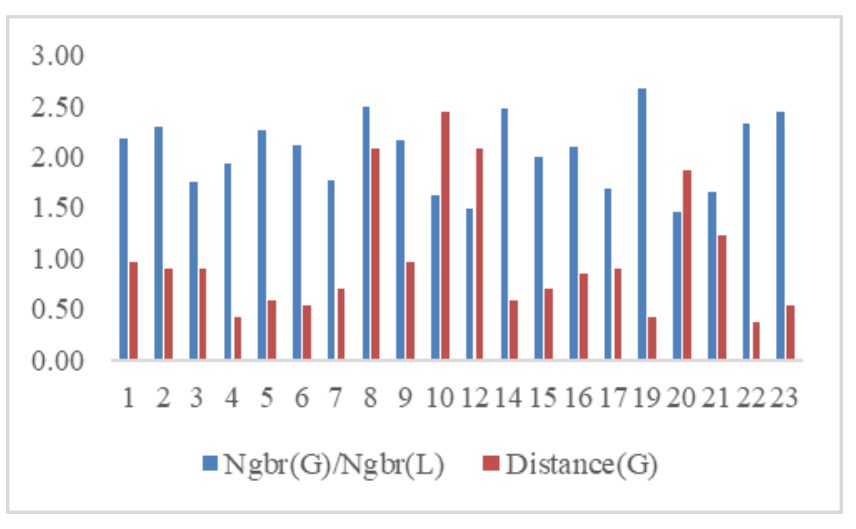

Figure 6: Analysis of Neighbors and Distances

Figure 6 represents the ratio of the number of neighbors between local and global DTMs. The higher the ratio is the more neighbors the individual has with increasing granularity. This observation confirmed that our models captured the individual characteristics that expressed differently in various social circumstances. The range of this ratio was observed from 1.447 to 2.67 with an average of 2.04 . P12 had the smallest ratio while P19 had the largest. In a sense, we interpreted that P19 had the biggest potential to make more neighbors by joining others. We also computed the ratio between the individual distance to all others and the average of all distances. By our definition, once a 
trajectory can be reachable by any cognitive state of another trajectory, the distance between these two trajectories becomes 0 . This can be another key to assess an individual's capability to communicate with others in varying situations. P22 had the smallest ratio of 0.37 , and $\mathrm{P} 10$ has the largest ratio of 2.447 with an average of 1 .

4.2.6 A Closer Look on P1. We have examined how individuals were presented variously in global and local DTMs for all 20 participants. The number of trajectories in each local SCOUT DTM was 2 for all, and the global DTM had 40 trajectories. In order to contemplate the change of individuals' trajectories in multiple granularity DTMs, we conducted another set of experiments, where the granularity of DTM was controlled by adding one more SCOUT subject at a time. We considered five participants (P1, P2, P3, P4, and P5) and built five DTMs from P1 (for DTM P1), a set of P1 and P2 (for DTM P12), a set of P1, P2 and P3 (for DTM P123), a set of P1, P2, P3 and P4 (for DTM P1234), and a set of P1, P2, P3, P4, and P5 (for DTM P12345).

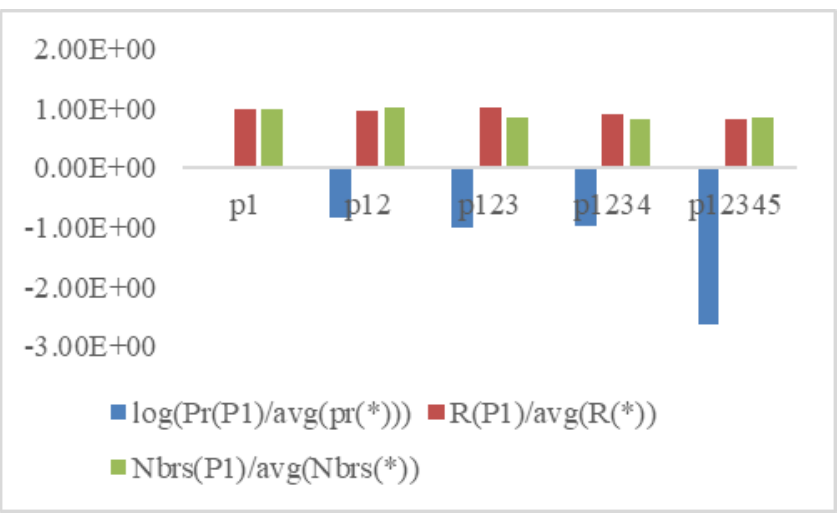

Figure 7: P1's change with DTM granularity

We were interested to see how individual P1 would be located in varying DTMs. We compared the trajectory probability of $\mathrm{P} 1$ against the average trajectory probability embedded in each DTM. The probability of trajectories of P1 changes from $3.19 \mathrm{E}-08 \rightarrow 9.59 \mathrm{E}-09 \rightarrow 1.07 \mathrm{E}-09 \rightarrow 2.08 \mathrm{E}-10 \rightarrow 1.18 \mathrm{E}-$ 12 while the average of all trajectories becomes $3.19 \mathrm{E}-08 \rightarrow 6.63 \mathrm{E}-$ $08 \rightarrow 1.07 \mathrm{E}-08 \rightarrow 1.97 \mathrm{E}-09 \rightarrow 5.09 \mathrm{E}-10$ respectively while comparing DTMs from P1 to P12345. The logarithmic difference between P1 and the average is presented in Figure 7. P1 was identified at the center at first but moved away from the center as more individuals were included. However, the distance between P1 and the average did not monotonically increase. From P123 to P1234, the distance became smaller while it was increased with an increasing number of trajectories involved in other cases.

The change of the ratio between the trajectory reward of P1 was more irregular than that of trajectory probability. The trajectory reward was identified to be independent of the granularity of DTMs and at a much smaller scale than that of trajectory probabilities. The number of P1 neighbors was observed to increase with the increasing granularity of DTMs while the average number of neighbors associated with all trajectories does not change in the same manner. By comparing the number of neighbors between P1 trajectories and others, we can infer the potential connectivity of $\mathrm{P} 1$, but the monotonic tendency was not observed.

\section{Conclusions}

In this paper, we investigated the human decision-making process and validated our computational models by answering questions associated with individuals' cognitive styles and other characteristics. Since we believe that individuals might not be associated with a particular cognitive style, we extend our earlier findings to more sophisticated hybrid decision-making styles and link them to understand the SCOUT subjects. For individual characteristics, we gathered the information representing riskaversion, selection utility, and game performance from the SCOUT raw data, and conducted the correlation analysis between the individual characteristics and the trajectory statistics of the DTMs developed from SCOUT dataset. We confirmed that individual characteristics could be properly represented with DTMs. Furthermore, we compared trajectory statistics including the probability and reward distribution in multiple DTMs as a way to consider granularity and confirmed that the decision-making process in various social conditions could be described properly through these DTMs.

\section{ACKNOWLEDGMENTS}

This work was sponsored in part by ONR Grant No. N00014-151-2154 and N00014-19-1-2211, AFOSR Grant No. FA9550-15-10383 and DURIP Grant No. N00014-15-1-2514.

\section{REFERENCES}

[1] AM. Parker and B. Fischhoff. 2005. Decision-making Competence: External Validation through an Individual-differences Approach. Journal of Behavioral Decision Making, 18, 1-27

[2] SG. Scott and RA Bruce. 1995. Decision-making style: The development and assessment of a new measure, Educational and Psychological Measurement, 55(5), 818-831

[3] F. Yu and. E. Santos Jr. 2016. On Modeling the Interplay between Opinion Change and Formation, in 29th Intl. FLAIRS Conference, Key Largo, FL

[4] E. Santos Jr. et al. 2017. Capturing a Commander's decision making style, in SPIE Defense + Security, Anaheim, California, United States

[5] C. Sibley et al. 2016. Demonstrating the Supervisory Control Operations User Testbed (SCOUT), Proceedings of the Human Factors and Ergonomics Society 2016 Annual Meeting, 60(1), 1324-1328

[6] E. Santos Jr. et al. 2018. Contextual Decision-Making Framework in Computational Context, W. Lawless, Ed., CRC Press

[7] R. Bellman. 1957. A Markovian Decision Process, Ind. Univ. Math. J, 679-684

[8] AY. Ng and SJ. Russell. 2000. Algorithms for Inverse Reinforcement Learning, in Proceedings of the $17^{\text {th }}$ Intl. Conf. on Machine Learning, San Francisco, CA, USA

[9] JC. Cox and GW. Harrison. 2008. Risk aversion in experiments: An introduction, 12, Emerald Group Publishing Limited, 1-7

[10] S. Bringsjord. 2008. Declarative/logic-based computational cognitive modeling, in The handbook of computational cognitive modeling, Cambridge University Press.

[11] S. Franklin et al. 2014. LIDA: A Systems-level Architecture for Cognition, Emotion, and Learning, 6(1), March.

[12] D. Silver et al. 2017. Mastering the game of go without human knowledge. Nature. Volume 550, pp. 354-359. October 19.

[13] M. Hutson. 2018. Basic instincts. Science Vol. 360(6391), pp. 845-847

[14] P. Wang, J. Rowe, W. Min, B. Mott, and J. Lester. 2017. Interactive narrative personalization with deep reinforcement learning. In Proceedings of the IJCAI'17, Carles Sierra (Ed.). AAAI Press 3852-3858. 